\title{
Bi-level CPAP does not improve gas exchange when compared with conventional CPAP for the treatment of neonates recovering from respiratory distress syndrome
}

\author{
Andrea L Lampland, ${ }^{1,2}$ Brenda Plumm, ${ }^{1}$ Cathy Worwa, ${ }^{1}$ Patricia Meyers, ${ }^{1}$ \\ Mark C Mammel ${ }^{1,2}$
}

${ }^{1}$ Infant Diagnostic \& Research Center, Children's Hospitals and Clinics of Minnesota, St Paul, Minnesota, USA ${ }^{2}$ Department of PediatricsNeonatology, University of Minnesota, Minneapolis, Minnesota, USA

\section{Correspondence to}

Dr Andrea L Lampland, Children's Hospitals and Clinics of Minnesota, 347 N. Smith Ave., Ste. 505, St. Paul, MN 55102, USA; lampl002@umn.edu

Received 13 November 2013 Revised 24 June 2014 Accepted 25 June 2014 Published Online First 12 July 2014

\section{SLinked}

- http://dx.doi.org/10.1136/ fetalneonatal-2013-305830

- http://dx.doi.org/10.1136/

fetalneonatal-2014-306109

\section{CrossMark}

To cite: Lampland $A L$, Plumm B, Worwa $C$, et al. Arch Dis Child Fetal Neonatal Ed 2015;100 F31-F34.

\section{ABSTRACT}

Aim We hypothesised that short-term application of bilevel nasal continuous positive airway pressure CPAP (SiPAP) compared with conventional nasal CPAP (nCPAP) at the same mean airway pressure in infants with persistent oxygen need recovering from respiratory distress syndrome would improve $\mathrm{CO}_{2}$ removal with no change in oxygen requirement.

Design Non-blinded, randomised, observational fourperiod crossover study.

Setting/population Level III NICU; low-birthweight infants requiring CPAP and oxygen while recovering from respiratory distress syndrome.

Methods Infants requiring nasal CPAP for $>24 \mathrm{~h}$ prior to study enrolment, and fraction of inspired oxygen requirement $\left(\mathrm{FiO}_{2}\right)$ of $0.25-0.5$, were randomised to either nCPAP or SiPAP. A crossover design with four $1 \mathrm{~h}$ treatment periods was used such that each infant received both treatments twice. Oxygen saturations $\left(\mathrm{SaO}_{2}\right)$, transcutaneous $\mathrm{CO}_{2}\left(\mathrm{tc} \mathrm{CO}_{2}\right)$ and vital signs were monitored continuously. Polysomnographic recordings were analysed for apnoea, bradycardia and oxygen desaturation.

Results Twenty low-birthweight infants receiving 0.3 $\pm 0.04 \%$ supplemental oxygen on CPAP of $6 \mathrm{~cm} \mathrm{H}_{2} \mathrm{O}$ were studied at an average of 33 days of age $( \pm 23$ days, $\mathrm{SD})$. There were no differences in $\mathrm{tcCO}_{2}$ or other physiological parameters except mean blood pressure, which was lower during nCPAP $(52.3 \pm 8.3$ vs

$54.4 \pm 9.1 \mathrm{~mm} \mathrm{Hg} ; \pm S D ; p<0.01)$. No differences in short or prolonged apnoea, bradycardia or significant desaturation events were observed.

Conclusions At similar mean airway pressures, SiPAP does not improve $\mathrm{CO}_{2}$ removal, oxygenation or other studied physiological parameters with the exception of mean blood pressure, which was not clinically significant

Trial registration number NCT01053455.

\section{INTRODUCTION}

Respiratory distress syndrome (RDS) remains a leading cause of morbidity and mortality in premature infants. ${ }^{1}$ The cascade of events that typifies RDS and its long-term counterpart, chronic lung disease, is rooted in the intrinsic deficits of the premature lung as well as exacerbated by mechanical ventilation. ${ }^{2}{ }^{3}$ Non-invasive ventilatory strategies, such as nasal continuous positive airway pressure (nCPAP), minimise lung inflammation and injury

\section{What is already known on this topic}

Respiratory distress syndrome continues to cause major morbidity in premature infants.

- Non-invasive respiratory support techniques, such as nasal continuous positive airway pressure (CPAP) and nasal intermittent positive pressure ventilation (NIPPV), are viable treatment options for infants with respiratory distress.

- Controversy exists regarding potential benefits of the various nasal CPAP and NIPPV techniques that are currently available for use in neonatal care.

\section{What this study adds}

As synchronisation of SiPAP is unavailable in the USA, this study specifically compares clinically relevant, pragmatic short-term respiratory outcomes when using nasal continuous positive airway pressure (CPAP) versus unsynchronised bi-level CPAP.

- The cyclic nature of pressure delivery with bi-level CPAP confers no benefit in augmenting ventilation over standard nasal CPAP when mean airway pressure is not increased.

associated with mechanical ventilation. ${ }^{4}$ Avoidance of intubation and increased use of early nCPAP to treat RDS has been shown to decrease exposure to mechanical ventilation and decrease the duration of supplemental oxygen therapy. ${ }^{5-8}$ In babies that require intubation and mechanical ventilation, use of nCPAP at the time of extubation has also been shown to decrease extubation failure. ${ }^{9}$

Recent studies have documented that the use of non-invasive nasal intermittent mechanical ventilation (NIPPV), with short inspiratory times and adequate ventilation rates, can satisfactorily treat hypoventilation, apnoea of prematurity and potentially decrease extubation failure when compared with the use of nCPAP. ${ }^{10}{ }^{11}$ However, most published studies of NIPPV in neonates use a very broad definition to include any form of nCPAP that 

NIPPV is bi-level CPAP, where CPAP delivery systems cycle the positive airway pressure between two levels and allow patients to breathe throughout the respiratory cycle, with the potential to improve oxygenation and ventilation. However, there have been very few studies to date looking at the use of bi-level CPAP as compared with the standard use of nCPAP in premature infants with respiratory distress, and those that exist have differences in how the bi-level CPAP support is delivered. ${ }^{12-14}$ To gain clarity on this commonly used mode of NIPPV, we compared unsynchronised bi-level nasal CPAP (Infant Flow SiPAP System, CareFusion Corporation, San Diego, California, USA) with standard nasal CPAP in low-birthweight infants with respiratory distress.

\section{METHODS}

In this randomised, observational crossover study, patient eligibility was based on the following inclusion criteria: birth weight $<2500 \mathrm{~g}$, ongoing treatment of respiratory distress with nasal CPAP for $>24 \mathrm{~h}$ prior to study enrolment and fraction of inspired oxygen requirement $\left(\mathrm{FiO}_{2}\right)$ of $0.25-0.5$ to keep oxygen saturations $85-95 \%$ for a minimum of $1 \mathrm{~h}$ prior to initiation of the study. Exclusion criteria included $\mathrm{FiO}_{2}$ requirement of $<0.25$ or $>0.5$; active medical treatment for PDA or culture proven sepsis, congenital defects of the airway, lungs or oesophagus, congenital cyanotic heart defects, genetic syndromes, or postoperative recovery period of $<24 \mathrm{~h}$. This study was registered at http://www.clinicaltrials.gov (\#NCT01053455) and approved by the Children's Hospitals and Clinics of Minnesota Institutional Review Board.

After written informed consent was obtained, the patient was placed in the supine position with the mouth closed by the aid of a soft chinstrap per our NICU standard care. A transcutaneous carbon dioxide monitor as well as pneumocardiogram sensors were placed on the infant (SenTec Digital Monitoring System, Therwil, Switzerland; Philips SmartMonitor, Andover, Massachusetts, USA; SpaceLabs Inc, Redmond, Washington, USA). The patient was randomised by sealed envelope shuffle to a starting treatment mode of either nCPAP at $6 \mathrm{~cm} \mathrm{H}_{2} \mathrm{O}$ pressure or unsynchronised bi-level CPAP (SiPAP) with the upper and lower pressure levels set to deliver the same mean airway pressure $\left(6 \mathrm{~cm} \mathrm{H} \mathrm{H}_{2} \mathrm{O}\right)$ with a minimum difference in upper and lower pressures of $3 \mathrm{~cm} \mathrm{H} \mathrm{H}_{2} \mathrm{O}$. SiPAP was set with the upper pressure to be delivered for $1 \mathrm{~s}$ at a rate of 20 cycles to the upper pressure per minute. Hudson short binasal prongs (Hudson Respiratory Care, Temecula, California, USA) were always used. All support was delivered by the Infant Flow SiPAP System with a flow rate of $8-9 \mathrm{~L} / \mathrm{min}$ in either the CPAP or BiPhasic mode (CareFusion Corporation, San Diego, California, USA). Research personnel adjusted the $\mathrm{FiO}_{2}$ to attain a targeted oxygen saturation of $88-90 \%$. The patients were maintained in the usual thermoneutral environment throughout the study, and all prescribed therapies were performed as ordered by the primary care team.

At study initiation, the infant was started on the randomised starting mode of either nCPAP or SiPAP. The study consisted of four $1 \mathrm{~h}$ study blocks, alternating from the initial mode to the alternate mode twice. During each study block, transcutaneous $\mathrm{CO}_{2}$ levels $\left(\mathrm{tcCO}_{2}\right)$, heart rate, respiratory rate and oxygen saturations were measured continuously (data acquisition every second using SenTec Digital Monitoring System, Therwil, Switzerland; SpaceLabs Inc, Redmond, Washington, USA). Three-channel pneumocardiogram data, including heart rate, respiratory effort, $\mathrm{SaO}_{2}$ and chest wall impedance, were has an intermittent increase in applied pressure. One type of

collected continuously for each block along with a nursing event $\log$ of patient cares/events (data acquisition every second using Philips SmartMonitor, Andover, MA). Apnoeic episodes were defined as absence of thoracic impedance change for a minimum of $10 \mathrm{~s}$. Bradycardic episodes were defined as persistent heart rate $<80$ beats per minute for a minimum of $10 \mathrm{~s}$. Significant desaturation episodes were defined as persistent pulse oximetry values $<80 \%$ for a minimum of $10 \mathrm{~s}$. Manual blood pressures were taken with appropriate sized neonatal blood pressure cuff every $10 \mathrm{~min}$ throughout the study. The study ended when the patient completed the $4 \mathrm{~h}$ study or was terminated early if the patient developed any signs of intolerance during the study, including persistent tachypnoea (respiratory rate $>80$ breaths per minute for $>10$ consecutive minutes), an increase of $>50 \%$ in the number of episodes of apnoea or bradycardia compared with the prestudy baseline noted $1 \mathrm{~h}$ preceding study entry, or increased supplemental $\mathrm{FiO}_{2}>0.3$ from prestudy baseline.

To allow for equilibration, we grouped and analysed data points from the last $20 \mathrm{~min}$ of each treatment block. Pneumocardiogram data were independently analysed and scored by a polysomnographic technologist blinded to study group. A sample size of 17 was calculated to detect a mean difference of $3 \mathrm{mmHg} \mathrm{tcCO}_{2}$ based on a two-tailed $\mathrm{p}$ value of 0.05 , power of 0.9 and a within-patient SD of $2.5 \mathrm{~mm} \mathrm{Hg}$. Data were analysed using commercial statistical software (Graphpad Prism V.5.0a; Chicago, Illinois, USA). Paired two-tailed t tests were employed, and $\mathrm{p}$ values $<0.05$ were considered statistically significant.

\section{RESULTS}

Twenty low-birthweight $(<2500 \mathrm{~g})$ infants were enrolled (table 1$)$. Eleven mothers received antenatal betamethasone treatment prior to delivery. In total, 17 of the 20 infants were delivered by caesarean section. Also, 19 of the 20 infants had a history of previous endotracheal intubation, exogenous surfactant administration and mechanical ventilation prior to study enrolment. All infants received caffeine citrate therapy for treatment of apnoea of prematurity. The average supplemental oxygen requirement at time of randomisation was $\mathrm{FiO}_{2}$ of $0.3( \pm 0.04)$. During the SiPAP blocks, the setting for the average high pressure was $8.9 \mathrm{~cm} \mathrm{H}_{2} \mathrm{O}$ $\left( \pm 0.4 \mathrm{~cm} \mathrm{H} \mathrm{H}_{2} \mathrm{O}, \mathrm{SD}\right)$ and the average low pressure was $4.1 \mathrm{~cm}$ $\mathrm{H}_{2} \mathrm{O}\left( \pm 0.2 \mathrm{~cm} \mathrm{H}_{2} \mathrm{O}\right)$.

There were no differences in $\mathrm{tcCO}_{2}$ values among the two treatment groups. Physiological variables, including heart rate, oxygen saturation, supplemental oxygen needs and respiratory rate, were similar among treatment groups. Average diastolic and mean blood pressure values were significantly higher in the SiPAP group compared with the nCPAP group (table 2). There

Table 1 Patient demographics

\begin{tabular}{|c|c|c|c|}
\hline & Mean value & SD & Range \\
\hline Gestational age & $\begin{array}{l}26 \text { weeks } \\
5 \text { days }\end{array}$ & $\begin{array}{l} \pm 1 \text { week } \\
6 \text { days }\end{array}$ & $\begin{array}{l}24 \text { weeks } 3 \text { days- } \\
31 \text { weeks } 4 \text { days }\end{array}$ \\
\hline Days of life at study entry & 33 days & \pm 23 days & $2-76$ days \\
\hline Birth weight & $897 \mathrm{~g}$ & $\pm 259 \mathrm{~g}$ & $600-1600 \mathrm{~g}$ \\
\hline Weight at study entry & $1310 \mathrm{~g}$ & $\pm 373 \mathrm{~g}$ & $830-2325 \mathrm{~g}$ \\
\hline $\begin{array}{l}\text { Days on nCPAP before } \\
\text { study entry }\end{array}$ & 7.6 days & \pm 7.8 days & $1-37$ days \\
\hline
\end{tabular}


Table 2 Primary physiological and respiratory variables

\begin{tabular}{lcll}
\hline & CPAP & SiPAP & p Value \\
\hline Heart rate $(\mathrm{bpm})$ & $168.2( \pm 12.3)$ & $167.3( \pm 12.1)$ & 0.4 \\
$\mathrm{O}_{2}$ saturation $(\%)$ & $87.4( \pm 2.9)$ & $87.3( \pm 3.8)$ & 1.0 \\
Transcutaneous $\mathrm{CO}_{2}(\mathrm{~mm} \mathrm{Hg})$ & $54( \pm 7.1)$ & $53.2( \pm 6.4)$ & 0.9 \\
Respiratory rate $(\mathrm{brpm})$ & $47.9( \pm 9.5)$ & $47.6( \pm 9.9)$ & 0.5 \\
$\mathrm{FiO}_{2}(\%)$ & $30.1( \pm 4.8)$ & $29.4( \pm 4.3)$ & 1.0 \\
Systolic blood pressure $(\mathrm{mm} \mathrm{Hg})$ & $68.4( \pm 10.4)$ & $69.8( \pm 10.9)$ & 0.1 \\
Diastolic blood pressure $(\mathrm{mm} \mathrm{Hg})$ & $42.5( \pm 7.4)$ & $45.1( \pm 8.8)$ & $0.003^{* *}$ \\
Mean blood pressure $(\mathrm{mm} \mathrm{Hg})$ & $52.3( \pm 8.3)$ & $54.4( \pm 9.1)$ & $0.01^{* *}$ \\
\hline
\end{tabular}

Mean $\pm S D ; * * p$ value $<0.05$.

bpm, beats per minute; brpm, breaths per minute; CPAP, continuous positive airways pressure.

were no significant differences in short (10-19s) or prolonged ( $\geq 20 \mathrm{~s}$ ) apnoeic, bradycardic or desaturation events between the two treatment groups (table 3 ).

\section{DISCUSSION}

In this randomised crossover trial, we compared two strategies for non-invasive support in babies recovering from RDS. Nasal CPAP is a strategy intended to recruit and stabilise lung volumes, and by doing so improve the mechanical behaviour of the lung. Bi-level CPAP is a newer non-invasive respiratory support technique that is essentially CPAP delivered at different pressures in a time-cycled manner, with ordered settings that are termed similarly to those used during invasive mechanical ventilation. In bi-level CPAP, the upper pressure settings are much lower than typical peak inspiratory pressures used with invasive mechanical ventilation and the bi-level CPAP inspiratory times are often much longer than those typically used with mechanical ventilation. This short-term crossover study was designed to test a simple physiological hypothesis that bi-level CPAP, administered at the same mean airway pressure as nCPAP, would improve ventilation and lower $\mathrm{CO}_{2}$, as reflected in $\mathrm{tcCO}_{2}$ values due to the cyclic pressure changes intended to augment minute ventilation.

In this study, we saw no evidence of improved ventilation during bi-level CPAP as the $\mathrm{tcCO}_{2}$ values were not different between the two treatment groups. Choosing a sample size adequate to detect a $3 \mathrm{~mm} \mathrm{Hg}$ change in $\mathrm{tcCO}_{2}$ with $90 \%$ power ensured that any meaningful change, if present, could have been detected. We also found no evidence that bi-level CPAP significantly impacted oxygenation, apnoea, bradycardia or desaturation events. We did observe a statistically significant, but clinically unimportant, change in blood pressures, with slightly higher mean and diastolic blood pressure values

Table 3 Pneumogram analysis

\begin{tabular}{lrcl}
\hline & \multicolumn{1}{c}{ CPAP } & \multicolumn{1}{l}{ SiPAP } & p Value \\
\hline Apnoea $10-19 \mathrm{~s}$ & $1.1( \pm 3.7)$ & $1.2( \pm 3.1)$ & 0.9 \\
Bradycardia 10-19 s & $0( \pm 0.2)$ & $0.1( \pm 0.3)$ & 0.3 \\
Desaturation $10-19 \mathrm{~s}$ & $2.0( \pm 2.3)$ & $1.9( \pm 2.9)$ & 1.0 \\
Apnoea $\geq 20 \mathrm{~s}$ & $0.4( \pm 1.1)$ & $1.0( \pm 4.6)$ & 0.5 \\
Bradycardia $\geq 20 \mathrm{~s}$ & $0.03( \pm 0.2)$ & $0( \pm 0)$ & 0.3 \\
Desaturation $\geq 20 \mathrm{~s}$ & $2.7( \pm 4.4)$ & $2.9( \pm 5.0)$ & 0.7 \\
\hline Mean number of episodes per study block \pm SD. & & \\
CPAP, continuous positive airways pressure. & &
\end{tabular}

recorded during the bi-level CPAP treatment periods. We speculate that the mean and diastolic blood pressures were higher in the bi-level CPAP group due to the $10 \mathrm{~min}$ sampling interval during the study blocks. Potentially, transient positive end-expiratory pressure-induced changes in cardiac output and blood pressure could have resulted in these differences by chance. $^{15}$

The use of bi-level CPAP has gained wide acceptance in spite of limited information on its indications, efficacy and proper application. ${ }^{12-14} 16$ Three studies evaluating the use of bi-level CPAP in preterm infants have been published. All three studies describe their choice of settings for bi-level CPAP as empiric since optimal parameters of the bi-level pressures have not been investigated. Migliori and colleagues' non-blinded crossover study in 2005 is most similarly structured to our study. In contrast to our study, Migliori's study provided increased mean airway pressure during the bi-level CPAP treatment blocks and cycled to the upper-level pressure more frequently (30 times per minute for $0.5 \mathrm{~s}$ per cycle). They evaluated bi-level CPAP delivered by the Infant Flow Advance ventilator (Electro Medical Equipment, Ltd., Brighton, UK) to nasal CPAP in 20 lowbirthweight infants and found a significant improvement in gas exchange during the bi-level CPAP treatment periods. They hypothesise that their findings of improved oxygenation and ventilation are related to increased mean airway pressure and ventilator-induced increase in tidal volume during the bi-level CPAP blocks. However, it still begs the question of whether the biphasic nature of the bi-level CPAP produces these effects or whether application of nasal CPAP at higher mean airway pressure would result in similar improvements.

Two other more recent studies have attempted to evaluate short-term safety and efficacy of bi-level CPAP compared with standard nasal CPAP. In 2010, Lista and colleagues compared synchronised bi-level CPAP and standard nasal CPAP in infants 28-34 weeks' gestation with respiratory distress at $1 \mathrm{~h}$ of life. They found no differences in short-term markers of inflammation between the two treatments. Although not powered for long-term pulmonary endpoints, their study did find the infants treated with bi-level CPAP had a significantly shorter duration of respiratory support and supplemental oxygen need than the group randomised to standard CPAP. In 2012, O'Brien and colleagues performed a randomised controlled trial of bi-level CPAP versus standard nasal CPAP to facilitate sustained extubation in babies $\leq 1250 \mathrm{~g}$. Although the trial was stopped five infants short of reaching the calculated sample size, they found that the use of bi-level CPAP was as effective as, but not statistically better than, standard CPAP at aiding sustained extubation.

Previous meta-analysis data have concluded that nasal intermittent positive pressure ventilation (NIPPV), of which bi-level CPAP is a subtype, increases the effectiveness of nCPAP by preventing the need for reintubation. ${ }^{10}$ However, this analysis is based on only three trials, performed between 1999 and 2002, using synchronised forms of NIPPV. None used SiPAP; additionally, synchronised SiPAP is not approved for use in the USA. These studies do not address how NIPPV may impact gas exchange and produce its effects. A recent large multicentre randomised controlled trial compared nCPAP with NIPPV in 1009 infants. The study was designed to compare rates of death before 36 weeks adjusted age or survival with bronchopulmonary dysplasia, as well as a number of secondary outcomes. Contrary to the meta-analysis conclusions, this study found no evidence that NIPPV in extremely low-birthweight infants improved the primary outcome or any of the many secondary outcomes studied. ${ }^{17}$ The questions asked in this study were 
global rather than targeted physiology outcomes and might seem to make the data we present moot. However, NIPPV in this pragmatic study was delivered using a number of different techniques, including bi-level CPAP as well as synchronised and unsynchronised ventilator-derived NIPPV. The question remains whether any of these techniques studied individually, as we have done, might produce different results, either acutely or over the long term.

Limitations of our study include its small sample size, heterogeneity in patient age and length of time on nCPAP before entering the study. Our population was recovering from RDS and requiring moderate supplemental oxygen on nCPAP, but overall, they were stable. Our analysis of only short-term physiological outcomes prevents any conclusions about important longer-term problems. Though only 20 patients were studied, the crossover design is powerful and allowed easy assessment of our targeted short-term respiratory outcomes. The addition of other important short-term respiratory physiology assessments, such as measurements of infant work of breathing in each treatment mode, would add further information. We acknowledge that short-term physiology does not define long-term outcomes but is an important first step for understanding how and why new techniques might be useful.

We studied unsynchronised SiPAP. Just as synchronisation produces important differences during convention mechanical ventilation, there may be differences between unsynchronised and synchronised non-invasive techniques. ${ }^{18}$ However, little information is available and is conflicting as to whether synchronisation confers any significant benefit. ${ }^{19} 20$ We chose unsynchronised SiPAP since it is the only type of bi-level CPAP available in the USA and is commonly used in other parts of the world. We acknowledge that by keeping mean airway pressure the same between periods, we used SiPAP in a manner that may differ from some physicians' clinical practice; at least some physicians use SiPAP at higher mean airway pressures than that used during nCPAP. We chose to keep mean airway pressure the same to minimise potential changes in lung volumes and oxygenation that might result and to better identify if the variable nature of pressure exposure by SiPAP in and of itself allows for improved short-term respiratory outcomes.

In conclusion, we compared two non-invasive respiratory support techniques, nCPAP and SiPAP, in a randomised crossover study in babies requiring oxygen therapy and recovering from RDS. We hypothesised that, at similar mean airway pressures, SiPAP would lower $\mathrm{tcCO}_{2}$. There were no differences in $\mathrm{tcCO}_{2}$ values between the two treatment groups or in other studied variables except for blood pressure. We saw a small but significant change in mean and diastolic blood pressures during SiPAP. Using this technique, SiPAP provides no physiological benefit compared with nCPAP in acute gas exchange or respiratory stability.

Contributors ALL, BP, CW, PM and MCM made substantive contributions to designing and implementing this clinical study and agree to be accountable for all aspects of the work they have done. BP, CW and PM made substantial contributions to data acquisition and analysis, critically revising the manuscript and giving final approval to the manuscript. ALL and MCM made substantial contributions to data analysis and interpretation, drafting and critically revising the manuscript and approving the final manuscript.

Funding The Children's Hospitals and Clinics of Minnesota Internal Research Grant Program funded this study.

Competing interests $\mathrm{AL}$ is a clinical research steering committee member for Discovery Labs, Inc.

Ethics approval Children's Hospitals and Clinics of Minnesota IRB.

Provenance and peer review Not commissioned; externally peer reviewed.

\section{REFERENCES}

1 Stevens TP, Blennow M, Soll RF. Early surfactant administration with brief ventilation vs. selective surfactant and continued mechanical ventilation for preterm infants with or at risk for respiratory distress syndrome. Cochrane Database Syst Rev 2004;3:CD003063.

2 Fanaroff A, Martin J. Neonatal-perinatal medicine, 7th edn. St Louis, MO: Mosby-Year Book, Inc., 2001:1003.

3 van Kaam A. Lung-protective ventilation in neonatology. Neonatology 2011;99:338-41.

4 Jobe AH, Kramer BW, Moss TJ, et al. Decreased indicators of lung injury with continuous positive expiratory pressure in preterm lambs. Pediatr Res 2002;52:387-92.

5 Gittermann GK, Fusch C, Gittermann AR, et al. Early nasal continuous positive airway pressure treatment reduces the need for intubation in very low birth weight infants. Eur J Pediatr 1997;156:384-8.

6 Morley CJ, Davis PG, Doyle LW, et al. Nasal CPAP or intubation at birth for very preterm infants. N Engl J Med 2008;358:700-8.

7 SUPPORT study group of the Eunice Kennedy Shriver NICHD Neonatal Research Network. Early CPAP versus surfactant in extremely preterm infants. $N$ Engl J Med 2010;362:1970-9.

8 Dunn MS, Kaempf J, de Klerk A, et al. Randomized trial comparing 3 approaches to the initial respiratory management of preterm neonates. Pediatrics 2011;128:e1069-76.

9 Davis PG, Henderson-Smart DJ. Nasal continuous positive airway pressure immediately after extubation for preventing morbidity in preterm infants. Cochrane Database Syst Rev 2003;2:CD000143.

10 Davis PG, Lemyre $B$, de Paoli AG. Nasal intermittent positive pressure ventilation (NIPPV) versus nasal continuous positive airway pressure (NCPAP) for preterm neonates after extubation. Cochrane Database Syst Rev 2001;3:CD003212.

11 Lemyre B, Davis PG, de Paoli AG. Nasal intermittent positive pressure ventilation (NIPPV) versus nasal continuous positive airway pressure (NCPAP) for apnea of prematurity. Cochrane Database Syst Rev 2002;1:CD002272.

12 Migliori C, Motta M, Angeli A, et al. Nasal bi-level vs. continuous positive airway pressure in preterm infants. Pediatr Pulmonol 2005;40:426-30.

13 Lista G, Castoldi F, Fontana P, et al. Nasal continuous positive airway pressure (CPAP) versus bi-level nasal CPAP in preterm babies with respiratory distress syndrome: a randomised control trial. Arch Dis Child Fetal Neonatal Ed 2010;95:F85-9.

14 O'Brien K, Campbell C, Brown L, et al. Infant flow biphasic nasal continuous positive airway pressure (BP-NCPAP) vs. infant flow NCPAP for the facilitation of extubation in infants' $\leq 1250$ grams: a randomized controlled trial. BMC Pediatr 2012;12:43.

15 Luecke T, Pelosi P. Clinical review: positive end-expiratory pressure and cardiac output. Crit Care 2005;9:607-21.

16 Kieran EA, Walsh H, O'Donnell CPF. Survey of nasal continuous positive airways pressure (NCPAP) and nasal intermittent positive pressure ventilation (NIPPV) use in Irish newborn nurseries. Arch Dis Child Fetal Neonatal Ed 2011;96:F156.

17 Kirpalani $H$, Millar D, Lemyre $B$, et al. A trial comparing noninvasive ventilation strategies in preterm infants. N Engl J Med 2013;369:611-20.

18 Greenough A, Dimitriou G, Prendergast M, et al. Synchronized mechanical ventilation for respiratory support in newborn infants. Cochrane Database Syst Rev 2008; 1:CD000456.

19 Dumpa V, Katz K, Northrup V, et al. SNIPPV vs NIPPV: does synchronization matter? J Perinatol 2012;32:438-42.

20 Chang HY, Claure N, D'Ugard C, et al. Effects of synchronization during nasal ventilation in clinically stable preterm infants. Pediatr Res 2011;69:84-9. 